\title{
Optimal regular differential operators with variable coefficients and applications
}

Veli Shakhmurov ${ }^{*}$

\section{"Correspondence:}

veli.sahmurov@okan.edu.tr

Department of Mechanical Engineering, Okan University,

Akfirat, Tuzla, Istanbul 34959, Turkey

\begin{abstract}
In this paper, maximal regularity properties for linear and nonlinear high-order elliptic differential-operator equations with VMO coefficients are studied. For the linear case, the uniform coercivity property of parameter-dependent boundary value problems is obtained in $L^{p}$ spaces. Then, the existence and uniqueness of a strong solution of the boundary value problem for a high-order nonlinear equation are established. In application, the maximal regularity properties of the anisotropic elliptic equation and the system of equations with VMO coefficients are derived.
\end{abstract}

AMS Subject Classification: 58|10; 58I20; 35Bxx; 35Dxx; 47Hxx; 47Dxx

Keywords: differential equations with VMO coefficients; boundary value problems; differential-operator equations; maximal $L^{p}$ regularity; abstract function spaces; nonlinear elliptic equations

\section{Introduction}

The goal of the present paper is to study the nonlocal boundary value problems (BVPs) for parameter-dependent linear differential-operator equations (DOEs) with discontinuous top-order coefficients

$$
s a(x) u^{(2 m)}(x)+A(x) u(x)+\sum_{k=0}^{2 m-1} s^{\frac{k}{2 m}} A_{k}(x) u^{(k)}(x)+\lambda u(x)=f(x),
$$

and the nonlinear equation

$$
a(x) u^{(2 m)}(x)+B\left(x, u, u^{(1)}, \ldots, u^{(2 m-1)}\right) u(x)=F\left(x, u, u^{(1)}, \ldots, u^{(2 m-1)}\right)
$$

where $a$ is a complex-valued function, $s$ is a positive and $\lambda$ is a complex parameter; $A=A(x), A_{k}=A_{k}(x)$ are linear and $B$ is a nonlinear operator in a Banach space $E$. Here the principal coefficients $a$ and $A$ may be discontinuous. More precisely, we assume that $a$ and $A(\cdot) A^{-1}\left(x_{0}\right)$ belong to the operator-valued Sarason class $V M O$ (vanishing mean oscillation). Sarason class $V M O$ was at first defined in [1]. In the recent years, there has been considerable interest to elliptic and parabolic equations with $V M O$ coefficients. This is mainly due to the fact that $V M O$ spaces contain as a subspace $C(\bar{\Omega})$ that ensures the extension of $L_{p}$-theory of operators with continuous coefficients to discontinuous coefficients (see, e.g., [2-11]). On the other hand, the Sobolev spaces $W^{1, n}(\Omega)$ and $W^{\sigma, \frac{\sigma}{n}}(\Omega)$,

\section{Springer}

(c) 2013 Shakhmurov; licensee Springer. This is an Open Access article distributed under the terms of the Creative Commons Attribution License (http://creativecommons.org/licenses/by/2.0), which permits unrestricted use, distribution, and reproduction in any medium, provided the original work is properly cited. 
$0<\sigma<1$, are also contained in VMO. Global regularity of the Dirichlet problem for elliptic equations with $V M O$ coefficients has been studied in [2-4]. We refer to the survey [3], where excellent presentation and relations with similar results can be found concerning the regularizing properties of these operators in the framework of Sobolev spaces.

It is known that many classes of PDEs (partial differential equations), pseudo DEs (differential equations) and integro DEs can be expressed in the form of DOEs. Many researchers (see, e.g., [12-24]) investigated similar spaces of functions and classes of PDEs under a single DOE. Moreover, the maximal regularity properties of DOEs with continuous coefficients were studied, e.g., in $[12,14,18,19]$.

Here the equation with top-order $V M O$-operator coefficients is considered in abstract spaces. We will prove the uniform separability of the problem (1), i.e., we show that for each $f \in L^{p}(0,1 ; E)$, there exists a unique strong solution $u$ of the problem (1) and a positive constant $C$ depending only $p, E, m$ and $A$ such that

$$
\sum_{i=0}^{2 m} s^{\frac{i}{2 m}}|\lambda|^{1-\frac{i}{2 m}}\left\|u^{(i)}\right\|_{L^{p}(0,1 ; E)}+\|A u\|_{L^{p}(0,1 ; E)} \leq C\|f\|_{L^{p}(0,1 ; E)} .
$$

Note that the principal part of a corresponding differential operator is non self-adjoint. Nevertheless, the sharp uniform coercive estimate for the resolvent and Fredholmness are established. Then, the existence and uniqueness of the above nonlinear problem are derived. In application, we study maximal regularity properties of anisotropic elliptic equations in mixed $L^{p}$ spaces and systems (finite or infinite) of differential equations with $V M O$ coefficients in the scalar $L^{p}$ space.

Since (1) involves unbounded operators, it is not easy to get representation for the Green function and the estimate of solutions. Therefore we use the modern harmonic analysis elements, e.g., the Hilbert operators and the commutator estimates in $E$-valued $L^{p}$ spaces, embedding theorems of Sobolev-Lions spaces and semigroup estimates to overcome these difficulties. Moreover, we also use our previous results on equations with continuous leading coefficients and the perturbation theory of linear operators to obtain main assertions.

\section{Notations and background}

Throughout the paper, we set $E$ a Banach space and $\Omega \subset R^{n} . L^{p}(\Omega ; E)$ denotes the space of all strongly measurable $E$-valued functions that are defined on $\Omega$ with the norm

$$
\|f\|_{p}=\|f\|_{L^{p}(\Omega ; E)}=\left(\int_{\Omega}\|f(x)\|_{E}^{p} d x\right)^{\frac{1}{p}}, \quad 1 \leq p<\infty
$$

$B M O(E)$ (bounded mean oscillation, see $[25,26])$ is the space of all $E$-valued local integrable functions with the norm

$$
\sup _{B} \int_{B}\left\|f(x)-f_{B}\right\|_{E} d x=\|f\|_{*, E}<\infty
$$

where $B$ ranges in the class of the balls in $R^{n},|B|$ is the Lebesgue measure of $B$ and $f_{B}$ is the average $\frac{1}{|B|} \int_{B} f(x) d x$. 
For $f \in B M O(E)$ and $r>0$, we set

$$
\sup _{\rho \leq r} \int_{B}\left\|f(x)-f_{B}\right\|_{E} d x=\eta(r)
$$

where $B$ ranges in the class of balls with radius $\rho$.

We will say that a function $f \in B M O(E)$ is in $V M O(E)$ if $\lim _{r \rightarrow+0} \eta(r)=0$. We will call $\eta(r)$ the $V M O$ modulus of $f$.

Note that if $E=C$, where $C$ is the set of complex numbers, then $B M O(E)$ and $V M O(E)$ coincide with John-Nirenberg class $B M O$ and Sarason class $V M O$, respectively.

The Banach space $E$ is called a $U M D$-space if the Hilbert operator

$$
(H f)(x)=\lim _{\varepsilon \rightarrow 0} \int_{|x-y|>\varepsilon} \frac{f(y)}{x-y} d y
$$

is bounded in $L_{p}(R, E), p \in(1, \infty)$ (see, e.g., [27]). UMD spaces include, e.g., $L_{p}, l_{p}$ spaces and Lorentz spaces $L_{p q}, p, q \in(1, \infty)$.

Let

$$
S_{\varphi}=\{\lambda \in C,|\arg \lambda| \leq \varphi\} \cup\{0\}, \quad 0 \leq \varphi<\pi .
$$

A linear operator $A$ is said to be $\varphi$-positive (or positive) in a Banach space $E$ with bound $M>0$ if $D(A)$ is dense on $E$ and

$$
\left\|(A+\lambda I)^{-1}\right\|_{L(E)} \leq M(1+|\lambda|)^{-1}
$$

for $\lambda \in S_{\varphi}, \varphi \in(0, \pi], I$ is an identity operator in $E$ and $L(E)$ is the space of bounded linear operators in $E$. Sometimes $A+\lambda I$ will be written as $A+\lambda$ and denoted by $A_{\lambda}$. It is known $\left[28, \mathbb{\$ 1 . 1 5 . 1 ]}\right.$ that there exist fractional powers $A^{\theta}$ of the positive operator $A$. Let $E\left(A^{\theta}\right)$ denote the space $D\left(A^{\theta}\right)$ with the graphical norm

$$
\|u\|_{E\left(A^{\theta}\right)}=\left(\|u\|^{p}+\left\|A^{\theta} u\right\|^{p}\right)^{\frac{1}{p}}, \quad 1 \leq p<\infty,-\infty<\theta<\infty .
$$

Let $E_{1}$ and $E_{2}$ be two Banach spaces. A set $W \subset L\left(E_{1}, E_{2}\right)$ is called $R$-bounded (see [14, 23]) if there is a positive constant $C$ such that for all $T_{1}, T_{2}, \ldots, T_{m} \in W$ and $u_{1}, u_{2}, \ldots, u_{m} \in$ $E_{1}, m \in N$,

$$
\int_{0}^{1}\left\|\sum_{j=1}^{m} r_{j}(y) T_{j} u_{j}\right\|_{E_{2}} d y \leq C \int_{0}^{1}\left\|\sum_{j=1}^{m} r_{j}(y) u_{j}\right\|_{E_{1}} d y,
$$

where $\left\{r_{j}\right\}$ is a sequence of independent symmetric $\{-1,1\}$-valued random variables on $[0,1]$.

Let $S\left(R^{n} ; E\right)$ denote the Schwartz class, i.e., the space of all $E$-valued rapidly decreasing smooth functions on $R^{n}$. Let $F$ denote the Fourier transformation. A function $\Psi \in$ $L^{\infty}\left(R^{n} ; B\left(E_{1}, E_{2}\right)\right)$ is called a Fourier multiplier from $L_{p}\left(R^{n} ; E_{1}\right)$ to $L_{p}\left(R^{n} ; E_{2}\right)$ if the map $u \rightarrow \Lambda_{\Psi} u=F^{-1} \Psi(\xi) F u, u \in S\left(R^{n} ; E_{1}\right)$ is well defined and extends to a bounded linear operator

$$
\Lambda_{\Psi}: L_{p}\left(R^{n} ; E_{1}\right) \rightarrow L_{p}\left(R^{n} ; E_{2}\right) .
$$


The set of all multipliers from $L_{p}\left(R^{n} ; E_{1}\right)$ to $L_{p}\left(R^{n} ; E_{2}\right)$ will be denoted by $M_{p}^{p}\left(E_{1}, E_{2}\right)$. For $E_{1}=E_{2}=E$, it will be denoted by $M_{p}^{p}(E)$.

Let

$$
U_{n}=\left\{\beta=\left(\beta_{1}, \beta_{2}, \ldots, \beta_{n}\right) \in N^{n}: \beta_{k} \in\{0,1\}\right\} .
$$

Definition $1 \mathrm{~A}$ Banach space $E$ is said to be a space satisfying a multiplier condition if for any $\Psi \in C^{(n)}\left(R^{n} ; L(E)\right)$, the $R$-boundedness of the set $\left\{\xi^{\beta} D_{\xi}^{\beta} \Psi(\xi): \xi \in R^{n} \backslash 0, \beta \in U_{n}\right\}$ implies that $\Psi$ is a Fourier multiplier in $L_{p}\left(R^{n} ; E\right)$, i.e., $\Psi \in M_{p}^{p}(E)$ for any $p \in(1, \infty)$.

Definition 2 The $\varphi$-positive operator $A$ is said to be an $R$-positive in a Banach space $E$ if there exists $\varphi \in[0, \pi)$ such that the set

$$
L_{A}=\left\{A(A+\lambda)^{-1}: \lambda \in S_{\varphi}\right\}
$$

is $R$-bounded.

A linear operator $A(x)$ is said to be positive in $E$ uniformly in $x$ if $D(A(x))$ is independent of $x, D(A(x))$ is dense in $E$ and

$$
\left\|(A(x)+\lambda)^{-1}\right\| \leq M(1+|\lambda|)^{-1}
$$

for all $\lambda \in S(\varphi), \varphi \in[0, \pi)$.

Let $\sigma_{\infty}\left(E_{1}, E_{2}\right)$ denote the space of all compact operators from $E_{1}$ to $E_{2}$. For $E_{1}=E_{2}=E$, it is denoted by $\sigma_{\infty}(E)$. Assume $E_{0}$ and $E$ are two Banach spaces and $E_{0}$ is continuously and densely embedded into $E$. Let $m$ be a natural number. $W^{m, p}\left(\Omega ; E_{0}, E\right)$ (the socalled Sobolev-Lions type space) denotes a space of all functions $u \in L^{p}\left(\Omega ; E_{0}\right)$ possessing the generalized derivatives $D_{k}^{m} u=\frac{\partial^{m} u}{\partial x_{k}^{m}}$ such that $D_{k}^{m} u \in L^{p}(\Omega ; E)$ is endowed with the norm

$$
\|u\|_{W^{m, p}\left(\Omega ; E_{0}, E\right)}=\|u\|_{L^{p}\left(\Omega ; E_{0}\right)}+\sum_{k=1}^{n}\left\|D_{k}^{m} u\right\|_{L^{p}(\Omega ; E)}<\infty
$$

For $E_{0}=E$ the space $W^{m, p}\left(\Omega ; E_{0}, E\right)$ will be denoted by $W^{m, p}(\Omega ; E)$. It is clear to see that

$$
W^{m, p}\left(\Omega ; E_{0}, E\right)=W^{m, p}(\Omega ; E) \cap L^{p}\left(\Omega ; E_{0}\right) .
$$

Let $s$ be a positive parameter. We define in $W^{m, p}\left(\Omega ; E_{0}, E\right)$ the following parameterized norm:

$$
\|u\|_{W_{s}^{m, p}\left(\Omega ; E_{0}, E\right)}=\|u\|_{L^{p}\left(\Omega ; E_{0}\right)}+\sum_{k=1}^{n}\left\|s D_{k}^{m} u\right\|_{L^{p}(\Omega ; E)} .
$$

Function $u \in W^{2, p}\left(0,1 ; E(A), E, L_{k}\right)=\left\{u \in W^{2, p}(0,1 ; E(A), E), L_{k} u=0\right\}$ satisfying equation (1) a.e. on $(0,1)$ is said to be a solution of the problem $(1)$ on $(0,1)$.

From [21] we have the following theorem. 
Theorem $\mathbf{A}_{1}$ Suppose the following conditions are satisfied:

(1) $E$ is a Banach space satisfying the multiplier condition with respect to $p \in(1, \infty)$ and $A$ is an $R$-positive operator in $E$;

(2) $\alpha=\left(\alpha_{1}, \alpha_{2}, \ldots, \alpha_{n}\right)$ are $n$-tuples of nonnegative integer numbers such that

$$
\varkappa=\frac{|\alpha|}{m} \leq 1 \quad \text { and } \quad 0<\mu \leq 1-\varkappa \text {; }
$$

(3) $\Omega \in R^{n}$ is a region such that there exists a bounded linear extension operator from $W^{m, p}(\Omega ; E(A), E)$ to $W^{m, p}\left(R^{n} ; E(A), E\right)$.

Then the embedding

$$
D^{\alpha} W^{m, p}(\Omega ; E(A), E) \subset L^{p}\left(\Omega ; E\left(A^{1-\varkappa-\mu}\right)\right)
$$

is continuous and there exists a positive constant $C_{\mu}$ such that

$$
s^{\frac{|\alpha|}{m}}\left\|D^{\alpha} u\right\|_{L^{p}\left(\Omega ; E\left(A^{1-\varkappa-\mu}\right)\right)} \leq C_{\mu}\left[h^{\mu}\|u\|_{W_{s}^{m, p}(\Omega ; E(A), E)}+h^{-(1-\mu)}\|u\|_{L^{p}(\Omega ; E)}\right]
$$

for all $u \in W^{m, p}(\Omega ; E(A), E)$ and $0<h \leq h_{0}<\infty$.

Theorem $\mathrm{A}_{2}$ Suppose all conditions of Theorem $\mathrm{A}_{1}$ are satisfied. Assume $\Omega$ is a bounded region in $R^{n}$ and $A^{-1} \in \sigma_{\infty}(E)$. Then, for $0<\mu \leq 1-\varkappa$, the embedding

$$
D^{\alpha} W^{m, p}(\Omega ; E(A), E) \subset L^{p}\left(\Omega ; E\left(A^{1-\varkappa-\mu}\right)\right)
$$

is compact.

In a similar way as in [2, Theorem 2.1], we have the following result.

Lemma $\mathbf{A}_{1}$ Let $E$ be a Banach space and $f \in V M O(E)$. The following conditions are equivalent:

(1) $f \in V M O(E)$;

(2) $f$ is in the BMO closure of the set of uniformly continuous functions which belong to $V M O$;

(3) $\lim _{y \rightarrow 0}\|f(x-y)-f(x)\|_{*, E}=0$

For $f \in L^{p}(\Omega ; E), p \in(1, \infty), a \in L^{\infty}\left(R^{n}\right)$, consider the commutator operator

$$
H[a, f](x)=a(x) H f(x)-H(a f)(x)=\lim _{\varepsilon \rightarrow 0} \int_{|x-y|>\varepsilon,} \frac{[a(x)-a(y)]}{x-y} f(y) d y .
$$

Proof Indeed, we observe that if $f \in V M O(E)$ with $V M O$ modulus $\eta$, there exists a constant $C$ such that $\|f(x-y)-f(x)\|_{*, E} \leq C \eta(r)$ for $\|y\| \leq r$ so that the $E$-valued usual mollifiers converge to $f$ in the $B M O$ norm. More precisely, given $f \in V M O(E)$ with $V M O$ modulus $\eta(r)$, we can find a sequence of $E$-valued $C^{\infty}$ functions $\left\{f_{h}\right\}$ converging to $f$ in $E$-valued $B M O$ spaces as $h \rightarrow 0$ with $V M O$ moduli $\eta_{h}$ such that $\eta_{h} \leq \eta(r)$. In a similar way, other cases are derived.

From [26, Theorem 1] and [29, Corollary 2.7], we have the following. 
Theorem $\mathbf{A}_{3}$ Let $E$ be a UMD space and $a \in V M O \cap L^{\infty}\left(R^{n}\right)$. Then $H[a, f]$ is a bounded operator in $L^{p}(R ; E), p \in(1, \infty)$.

From Theorem $\mathrm{A}_{3}$ and the property (2) of Lemma $\mathrm{A}_{1}$, we obtain, respectively:

Theorem $\mathbf{A}_{4}$ Assume all conditions of Theorem $\mathrm{A}_{3}$ are satisfied. Also, let $a \in V M O \cap$ $L^{\infty}\left(R^{n}\right)$ and let $\eta$ be the VMO modulus of a. Then, for any $\varepsilon>0$, there exists a positive number $\delta=\delta(\varepsilon, \eta)$ such that

$$
\|H[a, f]\|_{L^{p}(0, r ; E)} \leq M \varepsilon\|f\|_{L^{p}(0, r ; E)}, \quad r \in(0, \delta) .
$$

Theorem $\mathbf{A}_{5}$ Let $E$ be a $U M D$ space, $p \in(1, \infty)$ and $A(\cdot)$ uniformly R-positive in E. Moreover, let $A(\cdot) A^{-1}\left(x_{0}\right) \in L_{\infty}(R ; L(E)) \cap B M O(L(E)), x_{0} \in R$. Then the following commutator operator is bounded in $L^{p}(R ; E)$ :

$$
\begin{aligned}
H[A, f](x) & =A(x) A^{-1}\left(x_{0}\right) H f(x)-H\left(A(x) A^{-1}\left(x_{0}\right) f\right)(x) \\
& =\lim _{\varepsilon \rightarrow 0} \int_{|x-y|>\varepsilon,} \frac{\left[A(x) A^{-1}\left(x_{0}\right)-A(y) A^{-1}\left(x_{0}\right)\right]}{x-y} f(y) d y .
\end{aligned}
$$

Note that singular integral operators in $E$-valued $L^{p}$ spaces were studied, e.g., in [30].

Theorem $\mathrm{A}_{6}$ Assume all conditions of Theorem $\mathrm{A}_{5}$ are satisfied and $\eta$ is a VMO modulus of $A(\cdot) A^{-1}\left(x_{0}\right)$.

Then, for any $\varepsilon>0$, there exists a positive number $\delta=\delta(\varepsilon, \eta)$ such that

$$
\|H[A, f]\|_{L^{p}\left(\Omega_{r} ; E\right)} \leq M \varepsilon\|f\|_{L^{p}\left(\Omega_{r} ; E\right)}, \quad r \in(0, \delta) .
$$

Consider the nonlocal BVP for parameter-dependent DOE with constant coefficients

$$
\begin{aligned}
& (L+\lambda) u=\operatorname{sau}^{(2 m)}(x)+(A+\lambda) u(x)=f(x), \quad x \in(0,1), \\
& L_{k} u=\sum_{i=0}^{v_{k}} s^{\mu_{i}}\left[\alpha_{k i} u^{(i)}(0)+\beta_{k i} u^{(i)}(1)\right]=f_{k}, \quad k=1,2, \ldots, 2 m,
\end{aligned}
$$

where $v_{k} \in\{0,1, \ldots, 2 m-1\}, a, \alpha_{k i}, \beta_{k i}$ are complex numbers, $\mu_{i}=\frac{i}{2 m}+\frac{1}{2 m p}, \theta_{k}=\frac{v_{k}}{2 m}+\frac{1}{2 m p}$, $s$ is a positive and $\lambda$ is a complex parameter; $A_{\lambda}=A+\lambda$ and $A$ is a linear operator in $E$. Let $\omega_{1}, \omega_{2}, \ldots, \omega_{2 m}$ be roots of the equation $a \omega^{2 m}+1=0,\left[v_{i j}\right]$ be a $2 m$-dimensional matrix and $\eta=\left|\left[v_{i j}\right]\right|$ be a determinant of the matrix $\left[v_{i j}\right]$, where

$$
\begin{aligned}
& v_{i j}=\alpha_{j}\left(-\omega_{i}\right)^{\nu_{j}}, \quad i=1,2, \ldots, m, \quad v_{i j}=\beta_{j} \omega_{i}^{\nu_{j}}, \quad i=m+1, m+2, \ldots, 2 m, \\
& \alpha_{k}=\alpha_{k m_{k}}, \quad \beta_{k}=\beta_{k m_{k}}, \quad k, j=1,2, \ldots, 2 m .
\end{aligned}
$$

It is known that (see, e.g., [24, $\mathbb{1} 1.15]$ ) if the operator $A$ is $\varphi$-positive in $E$, then operators $\omega_{k} s^{-\frac{1}{2 m}} A_{\lambda}^{\frac{1}{2 m}}, k=1,2, \ldots, 2 m$ generate the following analytic semigroups:

$$
\begin{array}{ll}
U_{1 \lambda s}(x)=\exp \left(x \omega_{1} s^{-\frac{1}{2 m}} A_{\lambda}^{\frac{1}{2 m}}\right), \quad U_{2 \lambda s}(x)=\exp \left(x \omega_{2} s^{-\frac{1}{2 m}} A_{\lambda}^{\frac{1}{2 m}}\right), \quad \ldots, \\
U_{m \lambda s}(x)=\exp \left(x \omega_{m} s^{-\frac{1}{2 m}} A_{\lambda}^{\frac{1}{2 m}}\right), \quad U_{m+1 \lambda s}(x)=\exp \left(-(1-x) \omega_{m+1} s^{-\frac{1}{2 m}} A_{\lambda}^{\frac{1}{2 m}}\right),
\end{array}
$$




$$
\begin{aligned}
& U_{m+2 \lambda s}(x)=\exp \left(-(1-x) \omega_{m+2} s^{-\frac{1}{2 m}} A_{\lambda}^{\frac{1}{2 m}}\right), \quad \ldots, \\
& U_{2 m \lambda s}(x)=\exp \left(-(1-x) \omega_{2 m} s^{-\frac{1}{2 m}} A_{\lambda}^{\frac{1}{2 m}}\right) \quad \text { for } \lambda \in S(\varphi) .
\end{aligned}
$$

Let

$$
E_{k}=(E(A), E)_{\theta_{k}, p}
$$

From [19, Theorem 1] and [22, Theorem 3.2], we obtain the following.

Theorem $\mathbf{A}_{7}$ Assume the following conditions are satisfied:

(1) $E$ is a Banach space satisfying the multiplier condition with respect to $p \in(1, \infty)$;

(2) $A$ is an $R$-positive operator in $E$ for $0 \leq \varphi<\pi$ and $\eta \neq 0$;

(3) $\left|\arg \omega_{j}-\pi\right| \leq \frac{\pi}{2}-\varphi, j=1,2, \ldots, m,\left|\arg \omega_{j}\right| \leq \frac{\pi}{2}-\varphi, j=m+1, \ldots, 2 m$ and $\frac{\lambda}{\omega_{j}} \in S(\varphi)$.

Then

(1) for $f \in L_{p}(0,1 ; E), f_{k} \in E_{k}, \lambda \in S(\varphi)$ and for sufficiently large $|\lambda|$, the problem (2) has a unique solution $u \in W^{2 m, p}(0,1 ; E(A), E)$. Moreover, the following coercive uniform estimate holds:

$$
\sum_{i=0}^{2 m} s^{\frac{i}{2 m}}|\lambda|^{1-\frac{i}{2 m}}\left\|u^{(i)}\right\|_{L^{p}(0,1 ; E)}+\|A u\|_{L^{p}(0,1 ; E)} \leq C\left[\|f\|_{L^{p}(0,1 ; E)}+\sum_{k=1}^{2 m}\left\|f_{k}\right\|_{E_{k}}\right] .
$$

(2) For $f_{k}=0$, the solution is represented as

$$
\begin{aligned}
u(x) & =\int_{0}^{1} G_{\lambda s}(x, y) f(y) d y, G_{\lambda s}(x, y) \\
& =\sum_{k=1}^{2 m} \sum_{j=1}^{2 m} \sum_{i=0}^{\nu_{k}}\left[B_{k i j}(\lambda)\left(s^{-1} A_{\lambda}\right)^{-\frac{1}{2 m}\left(2 m+v_{k}-i-1\right)} U_{j \lambda s}(x) U_{k \lambda s}(1-y)\right]+U_{0 \lambda s}(x-y),
\end{aligned}
$$

where $B_{k i j}(\lambda)$ are uniformly bounded operators in $E$ and

$$
U_{0 \lambda s}(x-y)=\left\{\begin{array}{l}
a^{-1}\left\{s^{1-\frac{1}{2 m}} A_{\lambda}^{-\left(1-\frac{1}{2 m}\right)} \sum_{i=1}^{m}(-1)^{2 m+i} P_{i}^{-1} U_{i \lambda s}(x-y), x \geq y\right\}, \\
-a^{-1}\left\{s^{1-\frac{1}{2 m}} A_{\lambda}^{-\left(1-\frac{1}{2 m}\right)} \sum_{i=m+1}^{2 m}(-1)^{2 m+i} P_{i}^{-1} U_{i \lambda s}(x-y), x \leq y\right\},
\end{array}\right.
$$

where

$$
P_{i}=\left(\omega_{i}-\omega_{1}\right) \cdots\left(\omega_{i}-\omega_{i-1}\right)\left(\omega_{i+1}-\omega_{i}\right) \cdots\left(\omega_{2 m}-\omega_{i}\right), \quad i=1,2, \ldots, 2 m
$$

Consider the BVP for DOE with variable coefficients

$$
\begin{aligned}
& s a(x) u^{(2 m)}(x)+(A(x)+\lambda) u(x)=f(x), \quad x \in(0,1), \\
& L_{k} u=\sum_{i=0}^{v_{k}} s^{\theta_{k}}\left[\alpha_{k i} u^{(i)}(0)+\beta_{k i} u^{(i)}(1)\right]=0, \quad k=1,2, \ldots, 2 m,
\end{aligned}
$$


where $a=a(x)$ is a complex-valued function, $m_{k} \in\{0,1, \ldots, 2 m-1\}, \alpha_{k i}, \beta_{k i}$ are complex numbers, $s$ is a positive and $\lambda$ is a complex parameter, $\theta_{k}=\frac{v_{k}}{2 m}+\frac{1}{2 m p}$ and $A(x)$ is a linear operator in $E$.

Let $\omega_{1}=\omega_{1}(x), \omega_{2}=\omega_{2}(x), \ldots, \omega_{2 m}=\omega_{2 m}(x)$ be roots of the equation $a(x) \omega^{2 m}+1=0$, $\left[v_{i j}\right]$ be a $2 m$-dimensional matrix and $\eta(x)=\left|\left[v_{i j}\right]\right|$ be a determinant of the function matrix $\left[v_{i j}\right]$, where

$$
\begin{aligned}
& v_{i j}=v_{i j}(x)=\alpha_{j}\left(-\omega_{i}\right)^{v_{j}}, \quad i=1,2, \ldots, m, \quad v_{i j}=\beta_{j} \omega_{i}^{v_{j}}, \quad i=m+1, m+2, \ldots, 2 m, \\
& \alpha_{k}=\alpha_{k m_{k}}, \quad \beta_{k}=\beta_{k m_{k}}, \quad k, j=1,2, \ldots, 2 m .
\end{aligned}
$$

In the next theorem, we consider the case when principal coefficients are continuous. The well-posedness of this problem occurs in studying of equations with $V M O$ coefficients. From [19, Theorem 3] and [22, Theorem 3.2], we get the following.

\section{Theorem $\mathbf{A}_{8}$ Suppose the following conditions are satisfied:}

(1) $E$ is a Banach space satisfying the multiplier condition with respect to $p \in(1, \infty)$;

(2) $\left|\arg \omega_{j}-\pi\right| \leq \frac{\pi}{2}-\varphi, j=1,2, \ldots, m,\left|\arg \omega_{j}\right| \leq \frac{\pi}{2}-\varphi, j=m+1, \ldots, 2 m$ and $\frac{\lambda}{\omega_{j}} \in S(\varphi)$ a.e. $x \in(0,1)$;

(3) $a \in C[0,1], a(0)=a(1)$ and $\eta(x) \neq 0$ for a.e. $x \in[0,1]$;

(4) $A(x)$ is a uniformly R-positive operator in $E$ and

$$
A(\cdot) A^{-1}\left(x_{0}\right) \in C([0,1] ; L(E)), \quad x_{0} \in(0,1), \quad A(0)=A(1) .
$$

Then, for $f \in L^{p}(0,1 ; E), \lambda \in S(\varphi)$ and for sufficiently large $|\lambda|$, there is a unique solution $u \in W^{2, p}(0,1 ; E(A), E)$ of the problem (4). Moreover, the following coercive uniform estimate holds:

$$
\sum_{i=0}^{2 m} s^{\frac{i}{2 m}}|\lambda|^{1-\frac{i}{2 m}}\left\|u^{(i)}\right\|_{L^{p}(0,1 ; E)}+\|A u\|_{L^{p}(0,1 ; E)} \leq C\|f\|_{L^{p}(0,1 ; E)}
$$

\section{DOEs with VMO coefficients}

Consider the principal part of the problem (1)

$$
\begin{aligned}
& (L+\lambda) u=s a(x) u^{(2 m)}(x)+(A(x)+\lambda) u(x)=f(x), \quad x \in(0,1), \\
& L_{k} u=\sum_{i=0}^{m_{k}} s^{\mu_{i}}\left[\alpha_{k i} u^{(i)}(0)+\beta_{k i} u^{(i)}(1)\right]=0, \quad k=1,2, \ldots, 2 m .
\end{aligned}
$$

Condition 1 Assume the following conditions are satisfied:

(1) $E$ is a $U M D$ space, $p \in(1, \infty)$;

(2) $a \in V M O \cap L^{\infty}(R), \eta_{1}$ is a $V M O$ modulus of $a$;

(3) $\left|\arg \omega_{j}-\pi\right| \leq \frac{\pi}{2}-\varphi, j=1,2, \ldots, m,\left|\arg \omega_{j}\right| \leq \frac{\pi}{2}-\varphi, j=m+1, \ldots, 2 m$ and $\frac{\lambda}{\omega_{j}} \in S(\varphi)$ for $0 \leq \varphi<\pi, \eta(x) \neq 0$ a.e. $x \in[0,1]$;

(4) $A(x)$ is a uniformly $R$-positive operator in $E$ and

$$
A(\cdot) A^{-1}\left(x_{0}\right) \in L_{\infty}(0,1 ; L(E)) \cap V M O(L(E)), \quad x_{0} \in(0,1) ;
$$


(5) $a(0)=a(1), A(0)=A(1)$ and $\eta_{2}$ is a $V M O$ modulus of $A(\cdot) A^{-1}\left(x_{0}\right)$.

First, we obtain an integral representation formula for solutions.

Lemma 1 Let Condition 1 hold and $f \in L^{p}(0,1 ; E)$. Then, for all solutions $u$ of the problem (5) belonging to $W^{2 m, p}(0,1 ; E(A), E)$, we have

$$
\begin{aligned}
u^{(v)}(x)= & \int_{0}^{1} \Gamma_{\nu \lambda s}(x, x-y)\left\{[a(x)-a(y)] u^{(2 m)}(y)\right. \\
& +[A(x)-A(y)] u(y)+f(y)\} d y+f(x), \\
A(x) u(x) & =\int_{0}^{1} \Gamma_{2 \lambda s}^{\prime}(x, x-y)\left\{\left[a\left(x_{0}\right)-a(y)\right] u^{(2)}(y)+[A(x)-A(y)] u(y)+f(y)\right\} d y+f(x),
\end{aligned}
$$

where

$$
\begin{aligned}
& \Gamma_{\nu \lambda s}(x, x-y) \\
& \quad=\sum_{k=0}^{2 m} \sum_{j=1}^{2 m} \sum_{i=0}^{v_{k}}\left[B_{k \nu i j}^{\prime}(\lambda)\left(s^{-1} A_{\lambda}\right)^{-\frac{1}{2 m}\left(2 m+v_{k}+\nu-i-1\right)} U_{j \lambda s}(x) U_{i \lambda s}(1-y)\right]+U_{\nu 0 \lambda s}(x-y) .
\end{aligned}
$$

Here $B_{k i j}^{\prime}(\lambda)$ are uniformly bounded operators and

$$
U_{0 \lambda s}(x-y)=\left\{\begin{array}{l}
a^{-1}\left\{s^{1-\frac{1+v}{2 m}} A_{\lambda}^{-\left(1-\frac{1-v}{2 m}\right)} \sum_{i=1}^{m}(-1)^{2 m+i}\left(\omega_{i}\right)^{v} P_{i}^{-1} U_{i \lambda s}(x-y), x \geq y\right\}, \\
-a^{-1}\left\{s^{1-\frac{1+v}{2 m}} A_{\lambda}^{-\left(1-\frac{1-v}{2 m}\right)} \sum_{i=m+1}^{2 m}(-1)^{2 m+v+i}\left(\omega_{i}\right)^{v} P_{i}^{-1} U_{i \lambda s}(x-y), x \leq y\right\},
\end{array}\right.
$$

and the expression $\Gamma_{2 \lambda}^{\prime}(x, x-y)$ is a scalar multiple of $\Gamma_{2 \lambda}(x, x-y)$.

Proof Consider the problem (5) for $a(x)=a\left(x_{0}\right)$ and $A(x)=A\left(x_{0}\right)$, i.e.,

$$
\begin{aligned}
& \left(L_{0}+\lambda\right) u=s a\left(x_{0}\right) u^{(2 m)}(x)+\left(A\left(x_{0}\right)+\lambda\right) u(x)=f(x), \quad x \in(0,1), \\
& L_{k} u=\sum_{i=0}^{v_{k}} s^{\mu_{i}}\left[\alpha_{k i} u^{(i)}(0)+\beta_{k i} u^{(i)}(1)\right]=0, \quad k=1,2, \ldots, 2 m .
\end{aligned}
$$

Let $u \in C^{(2 m)}([0,1] ; E(A))$ be a solution of the problem (7). Taking into account the equality $L_{0} u=\left(L_{0}-L\right) u+L u$ and Theorem $\mathrm{A}_{7}$, we get

$$
\begin{aligned}
& u^{(v)}(x)=\int_{0}^{1} \Gamma_{v \lambda s}(x, x-y)\left\{\left[a\left(x_{0}\right)-a(y)\right] u^{(2 m)}(y)+\left[A\left(x_{0}\right)-A(y)\right] u(y)+f(y)\right\} d y+f(x), \\
& A\left(x_{0}\right) u(x)=\int_{0}^{1} \Gamma_{2 \lambda s}^{\prime}(x, x-y)\left\{\left[a\left(x_{0}\right)-a(y)\right] u^{(2)}(y)\right. \\
& \left.+\left[A\left(x_{0}\right)-A(y)\right] u(y)+f(y)\right\} d y+f(x) .
\end{aligned}
$$

Setting $x=x_{0}$ in the above, we get (6) for $u \in C^{(2 m)}([0,1] ; E(A))$. Then a density argument and Theorem $\mathrm{A}_{3}$ give the conclusion for

$$
u \in W^{2 m, p}(0,1 ; E(A), E), \quad L_{k} u=0 .
$$


Consider the problem $(5)$ on $(0, b)$, i.e.,

$$
\begin{aligned}
& \left(L_{b}+\lambda\right) u=s a(x) u^{(2 m)}(x)+(A(x)+\lambda) u(x)=f(x), \quad x \in(0, b), \\
& L_{b k} u=\sum_{i=0}^{v_{k}} s^{\mu_{i}}\left[\alpha_{k i} u^{(i)}(0)+\beta_{k i} u^{(i)}(b)\right]=0, \quad k=1,2, \ldots, 2 m .
\end{aligned}
$$

Theorem 1 Suppose Condition 1 is satisfied. Then there exists a number $b \in(0,1)$ such that the following uniform coercive estimate holds:

$$
\sum_{i=0}^{2 m} s^{\frac{i}{2 m}}|\lambda|^{1-\frac{i}{2 m}}\left\|u^{(i)}\right\|_{L^{p}(0, b ; E)}+\|A u\|_{L^{p}(0, b ; E)} \leq C\left\|\left(L_{b}+\lambda\right) u\right\|_{L^{p}(0, b ; E)}
$$

for $u \in W^{2 m, p}(0, b ; E(A), E), \lambda \in S(\varphi)$ with large enough $|\lambda|$.

Proof By Lemma 1, for any solution $u \in W^{2 m, p}(0, b ; E(A), E)$ of the problem (8), we have

$$
\begin{aligned}
u^{(v)}(x)= & \int_{0}^{b} \Gamma_{v b \lambda s}(x, x-y)\left\{[a(x)-a(y)] u^{(2)}(y)\right. \\
& +[A(x)-A(y)] u(y)+f(y)\} d y+f(x),
\end{aligned}
$$

where

$$
\begin{aligned}
& \Gamma_{\nu b \lambda s}(x, x-y) \\
& \quad=\sum_{k=0}^{2 m} \sum_{j=1}^{2 m} \sum_{i=0}^{\nu_{k}}\left[B_{v k i j}(\lambda)\left(s^{-1} A_{\lambda}\right)^{-\frac{1}{2 m}\left(2+v_{k}+v-i-1\right)} U_{j \lambda}(x) U_{i \lambda}(b-y)\right]+U_{0 v \lambda s}(x-y) ;
\end{aligned}
$$

here $B_{k i j}(\lambda)$ are uniformly bounded operators, and

$$
U_{0 \nu \lambda s}(x-y)=\left\{\begin{array}{l}
a^{-1}\left\{s^{1-\frac{1+v}{2 m}} A_{\lambda}^{-\left(1-\frac{1-}{2 m}\right)} \sum_{i=1}^{m}(-1)^{2 m+i} P_{i}^{-1} U_{i \lambda s}(x-y), x \geq y\right\}, \\
-a^{-1}\left\{s^{1-\frac{1}{2 m}} A_{\lambda}^{-\left(1-\frac{1}{2 m}\right)} \sum_{i=m+1}^{2 m}(-1)^{2 m+i} P_{i}^{-1} U_{i \lambda s}(x-y), x \leq y\right\} .
\end{array}\right.
$$

Moreover, from (10) and (11), clearly, we get

$$
A u(x)=\int_{0}^{b} \Gamma_{b \lambda s}^{\prime}(x, x-y)\left\{[a(x)-a(y)] u^{(2)}(y)+[A(x)-A(y)] u(y)+f(y)\right\} d y,
$$

where the expression $\Gamma_{b \lambda}^{\prime}(x, x-y)$ differs from $\Gamma_{2 b \lambda}(x, x-y)$ only by a constant.

Consider the operators

$$
\begin{aligned}
& B_{0 \lambda} f=\int_{0}^{1} G_{b \lambda s}(x, y) f(y) d y, \quad B_{i \lambda s} f=\int_{0}^{b} \Gamma_{i b \lambda s}(x, x-y) f(y) d y, \\
& S_{i \lambda} u=\int_{0}^{b} \Gamma_{i b \lambda}(x, x-y)[a(x)-a(y)] u^{(2)}(y) d y, \\
& D_{i \lambda s} u=\int_{0}^{b} \Gamma_{i b \lambda s}(x, x-y)[A(x)-A(y)] u(y) d y, \quad i=0,1,2,
\end{aligned}
$$




$$
\begin{aligned}
& \Phi_{1 \lambda s} u=\int_{0}^{b} \Gamma_{b \lambda s}^{\prime}(x, x-y)[a(x)-a(y)] u^{(2)}(y) d y, \\
& \Phi_{2 \lambda} u=\int_{0}^{b} \Gamma_{b \lambda}^{\prime}(x, x-y)[A(x)-A(y)] u(y) d y .
\end{aligned}
$$

Since the operators $B_{0 \lambda}$ and $B_{1 \lambda}$ are regular on $L^{p}(0, b ; E)$, by using the positivity properties of $A$ and the analyticity of semigroups $U_{k \lambda s}(x)$ in a similar way as in [20, Theorem 3.1], we get

$$
\left\|B_{i \lambda} f\right\|_{L^{p}(0, b ; E)} \leq M|\lambda|^{-\frac{2 m-i}{2 m}}\|f\|_{L^{p}(0, b ; E)}, \quad i=0,1 .
$$

Since the Hilbert operator is bounded in $L^{p}(R ; E)$ for a $U M D$ space $E$, we have

$$
\left\|B_{2 \lambda} f\right\|_{L^{p}(0, b ; E)} \leq M\|f\|_{L^{p}(0, b ; E)} .
$$

Thus, by virtue of Theorems $\mathrm{A}_{4}, \mathrm{~A}_{6}$ and in view of (10)-(12) for any $\varepsilon>0$, there exists a positive number $b=b\left(\varepsilon, \eta_{1}, \eta_{2}\right)$ such that

$$
\begin{aligned}
& \left\|S_{i \lambda} u\right\|_{L^{p}(0, b ; E)} \leq M \varepsilon|\lambda|^{-\frac{2 m-i}{2 m}}\left\|u^{(2)}\right\|_{L^{p}(0, b ; E)}, \\
& \left\|D_{i \lambda} u\right\|_{L^{p}(0, b ; E)} \leq M \varepsilon|\lambda|^{-\frac{2 m-i}{2 m}}\left\|A\left(x_{0}\right) u\right\|_{L^{p}(0, b ; E)}, \quad i=0,1,2, \\
& \left\|\Phi_{1 \lambda} u\right\|_{L^{p}(0, b ; E)} \leq M \varepsilon\left\|u^{(2 m)}\right\|_{L^{p}(0, b ; E)^{\prime}}, \quad\left\|\Phi_{2 \lambda} u\right\|_{L^{p}(0, b ; E)} \leq M \varepsilon\left\|A\left(x_{0}\right) u\right\|_{L^{p}(0, b ; E)} .
\end{aligned}
$$

Hence the estimates (13)-(15) imply (9).

Theorem 2 Assume Condition 1 holds. Let $u \in W^{2 m, p}(0,1 ; E(A), E)$ be a solution of (4). Then, for sufficiently large $|\lambda|, \lambda \in S(\varphi)$, the following coercive uniform estimate holds:

$$
\sum_{i=0}^{2 m} s^{\frac{i}{2 m}}|\lambda|^{1-\frac{i}{2 m}}\left\|u^{(i)}\right\|_{L^{p}(0,1 ; E)}+\|A u\|_{L^{p}(0,1 ; E)} \leq C\left[\|(L+\lambda) u\|_{L^{p}(0,1 ; E)}+\|u\|_{L^{p}(0,1 ; E)}\right]
$$

Proof This fact is shown by covering and flattening argument, in a similar way as in Theorem $\mathrm{A}_{8}$. Particularly, by partition of unity, the problem is localized. Choosing diameters of supports for corresponding finite functions, by using Theorem 1, Theorems $\mathrm{A}_{4}, \mathrm{~A}_{6}, \mathrm{~A}_{7}$ and embedding Theorem $\mathrm{A}_{1}$ (see the same technique for DOEs with continuous coefficients $[18,19])$, we obtain the assertion.

Let $Q_{s}$ denote the operator in $L^{p}(0,1 ; E)$ generated by the problem (4) for $\lambda=0$, i.e.,

$$
D\left(Q_{s}\right)=W^{2 m, p}\left(0,1 ; E(A), E, L_{k}\right), \quad Q_{s} u=s a(x) u^{(2 m)}+A(x) u .
$$

Theorem 3 Assume Condition 1 holds. Then, for all $f \in L^{p}(0,1 ; E), \lambda \in S(\varphi)$ and for large enough $|\lambda|$, the problem (5) has a unique solution $u \in W^{2 m, p}(0,1 ; E(A), E)$. Moreover, the following coercive uniform estimate holds:

$$
\sum_{i=0}^{2 m} s^{\frac{i}{2 m}}|\lambda|^{1-\frac{i}{2 m}}\left\|u^{(i)}\right\|_{L^{p}(0,1 ; E)}+\|A u\|_{L^{p}(0,1 ; E)} \leq C\|f\|_{L^{p}(0,1 ; E)}
$$


Proof First, let us show that the operator $Q+\lambda$ has a left inverse. Really, it is clear to see that

$$
\|u\|_{L^{p}(0,1 ; E)}=\frac{1}{|\lambda|}\left[\left\|\left(Q_{s}+\lambda\right) u\right\|_{L^{p}(0,1 ; E)}+\left\|Q_{s} u\right\|_{L^{p}(0,1 ; E)}\right] .
$$

By Theorem $\mathrm{A}_{1}$ for $u \in W^{2 m, p}(0,1 ; E(A), E)$, we have

$$
\left\|Q_{s} u\right\|_{L^{p}(0,1 ; E)} \leq C\|u\|_{W_{s}^{2 m, p}(0,1 ; E(A), E)} .
$$

Then, by virtue of (16) and in view of the above relations, we infer for all $u \in W^{2 m, p}(0,1$; $E(A), E)$ and sufficiently large $|\lambda|$ that there is a small $\varepsilon$ and $C(\varepsilon)$ such that

$$
\begin{aligned}
& \sum_{i=0}^{2 m} \frac{i}{2 m}|\lambda|^{1-\frac{i}{2 m}}\left\|u^{(i)}\right\|_{L^{p}(0,1 ; E)}+\|A u\|_{L^{p}(0,1 ; E)} \\
& \quad \leq C\left[\left\|\left(Q_{s}+\lambda\right) u\right\|_{L^{p}(0,1 ; E)}+\varepsilon\|u\|_{W^{2, p}(0,1 ; E(A), E)}+C(\varepsilon)\left\|\left(Q_{s}+\lambda\right) u\right\|_{L^{p}(0,1 ; E)}\right] .
\end{aligned}
$$

In view of (18) for $u \in W^{2 m, p}(0,1 ; E(A), E)$, we get

$$
\sum_{i=0}^{2 m} s^{\frac{i}{2 m}}|\lambda|^{1-\frac{i}{2 m}}\left\|u^{(i)}\right\|_{L^{p}(0,1 ; E)}+\|A u\|_{L^{p}(0,1 ; E)} \leq C\left\|\left(Q_{s}+\lambda\right) u\right\|_{L^{p}(0,1 ; E)} .
$$

The estimate (19) implies that (4) has a unique solution and the operator $Q_{s}+\lambda$ has a bounded inverse in its rank space. We need to show that the rank space coincides with the all space $L^{p}(0,1 ; E)$. It suffices to prove that there is a solution $u \in W^{2 m, p}(0,1 ; E(A), E)$ for all $f \in L^{p}(0,1 ; E)$. This fact can be derived in a standard way, approximating the equation with a similar one with smooth coefficients $[18,19]$. More precisely, by virtue of $[23$, Theorem 3.4], UMD spaces satisfy the multiplier condition. Moreover, by part (2) of Lemma $\mathrm{A}_{1}$, given $a \in V M O$ with $V M O$ modules $\eta(r)$, we can find a sequence of mollifiers functions $\left\{a_{h}\right\}$ converging to $a$ in $B M O$ as $h \rightarrow 0$ with $V M O$ modulus $\eta_{h}$ such that $\eta_{h}(r) \leq \eta(r)$. In a similar way, it can be derived for the operator function $A(x) A^{-1}\left(x_{0}\right) \in V M O(L(E))$.

Result 1 Theorem 3 implies that the resolvent $\left(Q_{s}+\lambda\right)^{-1}$ satisfies the following sharp uniform estimate:

$$
\sum_{i=0}^{2 m} s^{\frac{i}{2 m}}|\lambda|^{1-\frac{i}{2 m}}\left\|\frac{d^{i}}{d x^{i}}\left(Q_{s}+\lambda\right)^{-1}\right\|_{L\left(L^{p}(0,1 ; E)\right)}+\left\|A\left(Q_{s}+\lambda\right)^{-1}\right\|_{L\left(L^{p}(0,1 ; E)\right)} \leq C
$$

for $|\arg \lambda| \leq \varphi, \varphi \in(0, \pi)$ and $s>0$.

The estimate (20) particularly implies that the operator $Q$ is uniformly positive in $L^{p}(0,1 ; E)$ and generates an analytic semigroup for $\varphi \in\left(\frac{\pi}{2}, \pi\right)$ (see, e.g., [29, \$1.14.5]).

Remark 1 Conditions $a(0)=a(1), A(0)=A(1)$ arise due to nonlocality of the boundary conditions (4). If boundary conditions are local, then the conditions mentioned above are not required any more. 
Consider the problem (1), where $L_{k} u$ is the same boundary condition as in (4). Let $O_{s}$ denote the differential operator generated by the problem (1). We will show the separability and Fredholmness of (1).

Theorem 4 Assume the following:

(1) Condition 1 holds;

(2) for any $\varepsilon>0$, there is $C(\varepsilon)>0$ such that for a.e. $x \in(0,1)$ and

$$
\left\|A_{k}(x) u\right\|_{E} \leq \varepsilon\|u\|_{(E(A), E)} \frac{k}{2 m, \infty}+C(\varepsilon)\|u\|, \quad u \in(E(A), E)_{\frac{1}{2}, \infty} .
$$

Then, for all $f \in L^{p}(0,1 ; E)$ and for large enough $|\lambda|, \lambda \in S(\varphi)$, there is a unique solution $u \in$ $W^{2 m, p}(0,1 ; E(A), E)$ of the problem (1) and the following coercive uniform estimate holds:

$$
\sum_{i=0}^{2 m} s^{\frac{i}{2 m}}|\lambda|^{1-\frac{i}{2 m}}\left\|u^{(i)}\right\|_{L^{p}(0,1 ; E)}+\|A u\|_{L^{p}(0,1 ; E)} \leq C\|f\|_{L^{p}(0,1 ; E)} .
$$

Proof It is sufficient to show that the operator $O_{s}+\lambda$ has a bounded inverse $\left(O_{s}+\lambda\right)^{-1}$ from $L^{p}(0,1 ; E)$ to $W^{2 m, p}(0,1 ; E(A), E)$. Put $O_{s} u=Q_{s} u+Q_{1} u$, where

$$
Q_{1} u=\sum_{k=0}^{2 m-1} s^{\frac{k}{2 m}} A_{k}(x) u^{(k)}(x), \quad u \in W^{2 m, p}\left(0,1 ; E(A), E, L_{k}\right) .
$$

By the second assumption and Theorem $\mathrm{A}_{1}$, there is a small $\varepsilon$ and $C(\varepsilon)$ such that

$$
\begin{aligned}
\left\|s^{\frac{k}{2 m}} A_{k} u^{(k)}\right\|_{L^{p}(0,1 ; E)} & \leq C\left\|s^{\frac{k}{2 m}} A^{1-\frac{k}{2 m}} u\right\|_{L^{p}(0,1 ; E)} \\
& \leq \varepsilon\|u\|_{W_{s}^{2 m, p}(0,1 ; E(A), E)}+C(\varepsilon)\|u\|_{L^{p}(0,1 ; E)} .
\end{aligned}
$$

By Theorem 3, the operator $Q_{s}+\lambda$ has a bounded inverse $\left(Q_{s}+\lambda\right)^{-1}$ from $L^{p}(0,1 ; E)$ to $W^{2 m, p}(0,1 ; E(A), E)$ for sufficiently large $|\lambda|$. So, (22) implies the following uniform estimate:

$$
\left\|Q_{1}\left(Q_{s}+\lambda\right)^{-1}\right\|_{L\left(L^{p}(0,1 ; E)\right)}<1
$$

It is clear to see that

$$
\left(O_{s}+\lambda\right)=\left[I+Q_{1}\left(Q_{s}+\lambda\right)^{-1}\right]\left(Q_{s}+\lambda\right), \quad\left(O_{s}+\lambda\right)^{-1}=(Q+\lambda)^{-1}\left[I+Q_{1}\left(Q_{s}+\lambda\right)^{-1}\right]^{-1} .
$$

Then, by the above relation and by virtue of Theorem 3, we get the assertion.

Theorem 4 implies the following result.

Result 2 Suppose all conditions of Theorem 4 are satisfied. Then the resolvent $\left(O_{s}+\lambda\right)^{-1}$ of the operator $O_{s}$ satisfies the following sharp uniform estimate:

$$
\sum_{i=0}^{2 m} s^{\frac{i}{2 m}}|\lambda|^{1-\frac{i}{2 m}}\left\|\frac{d^{i}}{d x^{i}}\left(O_{s}+\lambda\right)^{-1}\right\|_{L\left(L^{p}(0,1 ; E)\right)}+\left\|A\left(O_{s}+\lambda\right)^{-1}\right\|_{L\left(L^{p}(0,1 ; E)\right)} \leq C
$$

for $|\arg \lambda| \leq \varphi, \varphi \in[0 \pi)$ and $s>0$. 
Consider the problem (1) for $\lambda=0$, i.e.,

$$
\begin{aligned}
& L u=s a(x) u^{(2 m)}(x)+A(x) u(x)+\sum_{k=0}^{2 m-1} s^{\frac{k}{2 m}} A_{k}(x) u^{(k)}(x)=f(x), \quad x \in(0,1), \\
& L_{k} u=\sum_{i=0}^{v_{k}} s^{\mu_{i}}\left[\alpha_{k i} u^{(i)}(0)+\beta_{k i} u^{(i)}(1)\right]=0, \quad k=1,2, \ldots, 2 m .
\end{aligned}
$$

Theorem 5 Assume all conditions of Theorem 4 hold and $A^{-1} \in \sigma_{\infty}(E)$. Then the problem (23) is Fredholm from $W^{2 m, p}(0,1 ; E(A), E)$ into $L^{p}(0,1 ; E)$.

Proof Theorem 4 implies that the operator $O_{s}+\lambda$ has a bounded inverse $\left(O_{s}+\lambda\right)^{-1}$ from $L^{p}(0,1 ; E)$ to $W^{2 m, p}(0,1 ; E(A), E)$ for large enough $|\lambda|$; that is, the operator $O_{s}+\lambda$ is Fredholm from $W^{2 m, p}(0,1 ; E(A), E)$ into $L^{p}(0,1 ; E)$. Then, by virtue of Theorem $\mathrm{A}_{2}$ and by perturbation theory of linear operators, we obtain the assertion.

\section{Nonlinear DOEs with VMO coefficients}

Let at first consider the linear BVP in a moving domain $(0, b(s))$

$$
\begin{aligned}
& L u=a(x) u^{(2 m)}(x)+A(x) u(x)+\sum_{k=0}^{2 m-1} A_{k}(x) u^{(k)}(x)=f(x), \quad x \in(0,1), \\
& L_{k} u=\sum_{i=0}^{v_{k}}\left[\alpha_{k i} u^{(i)}(0)+\beta_{k i} u^{(i)}(b)\right]=0, \quad k=1,2, \ldots, 2 m,
\end{aligned}
$$

where $a$ is a complex-valued function and $A=A(x), A_{k}=A_{k}(x)$ are linear operators in a Banach space $E$, where $b(s)$ is a positive continuous function independent of $u$.

Theorem 4 implies the following.

Result 3 Let all conditions of Theorem 4 be satisfied. Then the problem (24) has a unique solution $u \in W^{2 m, p}(0, b ; E(A), E)$ for $f \in L^{p}(0, b(s) ; E), p \in(1, \infty), \lambda \in S_{\varphi}$ with large enough $|\lambda|$, and the following coercive uniform estimate holds:

$$
\sum_{i=0}^{2 m}|\lambda|^{1-\frac{i}{2 m}}\left\|u^{(i)}\right\|_{L^{p}(0, b ; E)}+\|A u\|_{L^{p}(0, b ; E)} \leq\|f\|_{L^{p}(0, b ; E)} .
$$

Proof Really, under the substitution $\tau=x b(s)$, the moving boundary problem (24) maps to the following BVP with parameter in the fixed domain $(0,1)$ :

$$
\begin{aligned}
& b^{-2 m}(s) \tilde{a}(\tau) u^{(2 m)}(\tau)+(\tilde{A}+\lambda) u(\tau)+\sum_{k=0}^{2 m-1} b^{-k}(s) \tilde{A}_{k}(\tau) u^{(k)}(\tau)=\tilde{f}(\tau), \\
& \sum_{i=0}^{m_{j}} b^{-i}(s)\left[\alpha_{j i} u^{(i)}(0)+\beta_{j i} u^{(i)}(1)\right]=0, \quad j=1,2, \ldots, 2 m,
\end{aligned}
$$

where

$$
\tau \in(0,1), \quad \tilde{A}=A\left(\tau b^{-1}(s)\right), \quad \tilde{A}_{k}=A_{k}\left(\tau b^{-1}(s)\right), \tilde{f}(\tau)=f\left(\tau b^{-1}(s)\right) .
$$

Then, by virtue of Theorem 4, we obtain the assertion. 
Consider the following nonlinear problem:

$$
\begin{aligned}
& a(x) u^{(2 m)}(x)+B\left(x, u, u^{(1)}, \ldots, u^{(2 m-1)}\right) u(x)=F\left(x, u, u^{(1)}, \ldots, u^{(2 m-1)}\right), \\
& \sum_{i=0}^{\nu_{k}}\left[\alpha_{k i} u^{(i)}(0)+\beta_{k i} u^{(i)}(b)\right]=0, \quad k=1,2, \ldots, 2 m,
\end{aligned}
$$

where $v_{k} \in\{0,1, \ldots, 2 m-1\}, \alpha_{k i}, \beta_{k i}$ are complex numbers, $x \in(0, b)$, where $b$ is a positive number in $\left(0, b_{0}\right]$.

In this section, we will prove the existence and uniqueness of a maximal regular solution of the nonlinear problem (25). Assume $A$ is a $\varphi$-positive operator in a Banach space $E$. Let

$$
\begin{array}{ll}
X=L^{p}(0, b ; E), & Y=W^{2 m, p}(0, b ; E(A), E), \\
E_{i}=(E(A), E)_{\sigma_{i}, p}, & \sigma_{i}=\frac{1+i p}{2 m p}, \quad X_{0}=\prod_{i=0}^{2 m-1} E_{j} .
\end{array}
$$

Remark 2 By using [28, $\mathbb{1} 1.8$.], we obtain that the embedding $D^{j} Y \in E_{j}$ is continuous and there exists a constant $C_{1}$ such that for $w \in Y, W=\left\{w_{0}, w_{1}, \ldots, w_{2 m-1}\right\}, w_{j}=D^{j} w(\cdot)$,

$$
\|w\|_{X_{0}, \infty}=\prod_{i=0}^{2 m-1}\left\|D^{i} w\right\|_{C\left([0, b], E_{j}\right)}=\sup _{x \in[0, b]} \prod_{i=0}^{1}\left\|D^{i} w(x)\right\|_{E_{j}} \leq C_{1}\|w\|_{Y} .
$$

Condition 2 Assume the following are satisfied:

(1) $E$ is a $U M D$ space, $p \in(1, \infty)$;

(2) $a \in V M O \cap L^{\infty}(R), a(0)=a(b)$;

(3) $\left|\arg \omega_{j}-\pi\right| \leq \frac{\pi}{2}-\varphi, j=1,2, \ldots, m,\left|\arg \omega_{j}\right| \leq \frac{\pi}{2}-\varphi, j=m+1, \ldots, 2 m$ and $\frac{\lambda}{\omega_{j}} \in S(\varphi)$ for $\lambda \in S(\varphi), 0 \leq \varphi<\pi, \eta(x) \neq 0$ a.e. $x \in[0,1]$;

(3) $F\left(x, v_{0}, v_{1}, \ldots, v_{2 m-1}\right):[0, b] \times X_{0} \rightarrow E$ is a measurable function for each $v_{i} \in E_{i}$, $i=0,1, \ldots, 2 m-1 ; F(x, \cdot, \cdot)$ is continuous with respect to $x \in[0, b]$ and $f(x)=F(x, 0) \in X$. Moreover, for each $R>0$, there exists $\mu_{R}$ such that

$$
\|F(x, U)-F(x, \bar{U})\|_{E} \leq \mu_{R}\|U-\bar{U}\|_{X_{0}}
$$

where $U=\left\{u_{0}, u_{1}, \ldots, u_{2 m-1}\right\}$ and $\bar{U}=\left\{\bar{u}_{0}, \bar{u}_{1}, \ldots, \bar{u}_{2 m-1}\right\}$ for a.a. $x \in[0, b], u_{i}, \bar{u}_{i} \in E_{i}$ and

$$
\|U\|_{X_{0}} \leq R,\|\bar{U}\|_{X_{0}} \leq R
$$

(4) for $U \in X_{0}$, the operator $B(x, U)$ is $R$-positive in $E$ uniformly with respect to $x \in[0, b] ; B(x, U) B^{-1}\left(x^{0}, U\right) \in L_{\infty}(0,1 ; L(E)) \cap V M O(L(E)), x_{0} \in(0,1)$, where domain definition $D(B(x, U))$ does not depend on $x$ and $U ; B(x, W):(0, b) \times X_{0} \rightarrow B(E(A), E)$ is continuous, where $A=A(x)=B(x, W)$ for fixed $W=\left\{w_{0}, w_{1}, \ldots, w_{2 m-1}\right\} \in X_{0}$;

(5) for each $R>0$, there is a positive constant $L(R)$ such that $\|[B(x, U)-B(x, \bar{U})] v\|_{E} \leq L(R)\|U-\bar{U}\|_{X_{0}}\|A v\|_{E}$ for $x \in(0, b), U, \bar{U} \in X_{0}$, $\|U\|_{X_{0}},\|\bar{U}\|_{X_{0}} \leq R$ and $v \in D(A)$ and $A(0)=A(b)$. 
Theorem 6 Let Condition 2 hold. Then there is $b \in\left(0, b_{0}\right]$ such that the problem (26) has a unique solution belonging to space $W^{2 m, p}(0, b ; E(A), E)$.

Proof Consider the linear problem

$$
\begin{aligned}
& -a(x) w^{(2 m)}(x)+(A(x)+d) w(x)=f(x), \\
& L_{k} w=\sum_{i=0}^{v_{k}} \alpha_{k i} w^{(i)}(0)+\beta_{k i} w^{(i)}(b)=0, \quad k=1,2, \ldots, 2 m,
\end{aligned}
$$

where

$$
f(x)=F(x, 0), \quad x_{0} \in(0, b) .
$$

By virtue of Result 3, the problem (26) has a unique solution for all $f \in X$ and for sufficiently large $d>0$ that satisfies the following:

$$
\|w\|_{Y} \leq C_{0}\|f\|_{X}
$$

where the constant $C$ does not depend on $f \in X$ and $b \in\left(0, b_{0}\right]$. We want to solve the problem (25) locally by means of maximal regularity of the linear problem (26) via the contraction mapping theorem. For this purpose, let $w$ be a solution of the linear BVP (27). Consider a ball

$$
B_{r}=\left\{v \in Y, L_{k} v=0, k=1,2, \ldots, 2 m,\|v-w\|_{Y} \leq r\right\} .
$$

For $v \in B_{r}$, consider the linear problem

$$
\begin{aligned}
& -a(x) u^{(2 m)}(x)+A u(x)+d u=F(x, V)+[B(0, W)-B(x, V)] v, \\
& L_{k} u=\sum_{i=0}^{v_{k}} \alpha_{k i} u^{(i)}(0)+\beta_{k i} u^{(i)}(b)=0, \quad k=1,2, \ldots, 2 m,
\end{aligned}
$$

where

$$
V=\left\{v, v^{(1)}, \ldots, v^{(2 m-1)}\right\}, \quad W=\left\{w, w^{(1)}, \ldots, w^{(2 m-1)}\right\} .
$$

Define a map $Q$ on $B_{r}$ by $Q v=u$, where $u$ is a solution of the problem (27). We want to show that $Q\left(B_{r}\right) \subset B_{r}$ and that $Q$ is a contraction operator provided $b$ is sufficiently small and $r$ is chosen properly. For this aim, by using maximal regularity properties of the problem (28), we have

$$
\|Q v-w\|_{Y}=\|u-w\|_{Y} \leq C_{0}\left\{\|F(x, V)-F(x, 0)\|_{X}+\|[B(0, W)-B(x, V)] v\|_{X}\right\} .
$$

By assumption (5), we have

$$
\begin{aligned}
& \|[B(0, W) v-B(x, V)] v\|_{X} \\
& \quad \leq \sup _{x \in[0, b]}\left\{\|[B(0, W)-B(x, W)] v\|_{B\left(X_{0}, X\right)}\right.
\end{aligned}
$$




$$
\begin{aligned}
& \left.+\|B(x, W)-B(x, V)\|_{B\left(X_{0}, X\right)}\|v\|_{Y}\right\} \\
\leq & {\left[\delta(b)+L(R)\|W-V\|_{\infty, X_{0}}\right]\left[\|v-w\|_{Y}+\|w\|_{Y}\right] } \\
\leq & \left\{\delta(b)+L(R)\left[C_{1}\|v-w\|_{Y}+\|v-w\|_{Y}\right]\left[\|v-w\|_{Y}+\|w\|_{Y}\right]\right\} \\
\leq & \delta(b)+L(R)\left[C_{1} r+r\right]\left[r+\|w\|_{Y}\right],
\end{aligned}
$$

where

$$
\delta(b)=\sup _{x \in[0, b]}\|[B(0, W)-B(x, W)]\|_{B\left(X_{0}, X\right)} .
$$

Bear in mind

$$
\begin{aligned}
& \|F(x, V)-F(x, 0,)\|_{E} \\
& \quad \leq \delta(b)+\|F(x, V)-F(x, W)\|_{E}+\|F(x, W)-F(x, 0)\|_{E} \\
& \quad \leq \delta(b)+\mu_{R}\left[\|v-w\|_{Y}+\|w\|_{Y}\right] \mu_{R} C_{1}\left[\|v-w\|_{Y}+\|w\|_{Y}\right] \\
& \quad \leq \mu_{R}\left[C_{1} r+\|w\|_{Y}\right],
\end{aligned}
$$

where $R=C_{1} r+\|w\|_{Y}$ is a fixed number. In view of the above estimates, by a suitable choice of $\mu_{R}, L_{R}$ and for sufficiently small $b \in\left[0 ; b_{0}\right)$, we have

$$
\|Q v-w\|_{Y} \leq r
$$

i.e.,

$$
Q\left(B_{r}\right) \subset B_{r} .
$$

Moreover, in a similar way, we obtain

$$
\begin{aligned}
\|Q v-Q \bar{v}\|_{Y} \leq & C_{0}\left\{\mu_{R} C_{1}+M_{a}+L(R)\left[\|v-w\|_{Y}+C_{1} r\right]\right. \\
& \left.+L(R) C_{1}\left[r+\|w\|_{Y}\right]\|v-\bar{v}\|_{Y}\right\}+\delta(b) .
\end{aligned}
$$

By a suitable choice of $\mu_{R}, L_{R}$ and for sufficiently small $b \in\left(0, b_{0}\right)$, we obtain $\| Q v-$ $Q \bar{v}\left\|_{Y}<\eta\right\| v-\bar{v} \|_{Y}, \eta<1$, i.e., $Q$ is a contraction operator. Eventually, the contraction mapping principle implies a unique fixed point of $Q$ in $B_{r}$ which is the unique strong solution $u \in Y$.

\section{Boundary value problems for anisotropic elliptic equations with VMO coefficients}

The Fredholm property of BVPs for elliptic equations with parameters in smooth domains were studied, e.g., in [14, 24, 28]; also, for non-smooth domains, these questions were investigated, e.g., in [31].

Let $\Omega \subset R^{n}$ be an open connected set with compact $C^{2 l}$-boundary $\partial \Omega$. Let us consider the nonlocal boundary value problems on a cylindrical domain $G=(0,1) \times \Omega$ for the fol- 
lowing anisotropic elliptic equation with $V M O$ top-order coefficients:

$$
\begin{gathered}
\begin{aligned}
(L+\lambda) u= & s a(x) \frac{\partial^{2 m} u}{\partial x^{2 m}}+\sum_{k=0}^{2 m-1} s^{\frac{k}{2 m}} d_{k}(x, y) \frac{\partial^{k} u}{\partial x^{k}} \\
& +\sum_{|\alpha| \leq 2 l} a_{\alpha}(y) D_{y}^{\alpha} u+\lambda u=f(x, y), \quad x \in(0,1), y \in \Omega,
\end{aligned} \\
L_{k} u=\sum_{i=0}^{\nu_{k}} s^{\mu_{i}}\left[\alpha_{k i} u_{x}^{(i)}(0, y)+\beta_{k i} u_{x}^{(i)}(1, y)\right]=0, \quad k=1,2, \ldots, 2 m, \\
B_{j} u=\sum_{|\beta| \leq l_{j}} b_{j \beta}(y) D_{y}^{\beta} u(x, y)=0, \quad x \in(0,1), y \in \partial \Omega, j=1,2, \ldots, l,
\end{gathered}
$$

where $s$ is a positive parameter, $a, d_{i}$ are complex-valued functions, $\alpha_{k i}$ and $\beta_{k i}$ are complex numbers,

$$
D_{j}=-i \frac{\partial}{\partial y_{j}}, \quad v_{k} \in\{0,1, \ldots, 2 m-1\}, \quad y=\left(y_{1}, \ldots, y_{n}\right), \quad \mu_{i}=\frac{i}{2 m}+\frac{1}{2 m p} .
$$

For $G=(0,1) \times \Omega, \mathbf{p}=\left(p_{1}, p\right), L^{\mathbf{P}}(G)$ will denote the space of all $\mathbf{p}$-summable scalarvalued functions with a mixed norm (see, e.g., [32, $\$ 1]$ ), i.e., the space of all measurable functions $f$ defined on $G$, for which

$$
\|f\|_{L^{\mathbf{p}}(G)}=\left(\int_{0}^{1}\left(\int_{\Omega}|f(x, y)|^{p_{1}} d y\right)^{\frac{p}{p_{1}}} d x\right)^{\frac{1}{p}}<\infty
$$

Analogously, $W^{2 m, 2 l, \mathbf{p}}(G)$ denotes the anisotropic Sobolev space with the corresponding mixed norm $[32, \$ 10]$.

Theorem 7 Let the following conditions be satisfied:

(1) $a, d_{0} \in V M O \cap L^{\infty}(R), a(0)=a(1)$;

(2) $\left|\arg \omega_{j}-\pi\right| \leq \frac{\pi}{2}-\varphi, j=1,2, \ldots, m,\left|\arg \omega_{j}\right| \leq \frac{\pi}{2}-\varphi, j=m+1, \ldots, 2 m$ and $\frac{\lambda}{\omega_{j}} \in S(\varphi)$ for $\lambda \in S(\varphi), 0 \leq \varphi<\pi, \eta(x) \neq 0$, a.e. $x \in[0,1]$;

(3) $d_{k} \in L^{\infty}, d_{k}(\cdot, y) d_{0}^{1-\frac{k}{2 m}-\sigma_{k}}(\cdot) \in L^{\infty}(0,1)$ for a.e. $y \in \Omega$ and $0<\sigma_{k}<1-\frac{k}{2 m}$;

(4) $a_{\alpha} \in V M O \cap L^{\infty}\left(R^{n}\right)$ for each $|\alpha|=2 l$ and $a_{\alpha} \in\left[L^{\infty}+L^{\gamma_{k}}\right](\Omega)$ for each $|\alpha|=k<2 l$ with $r_{k} \geq q$ and $2 l-k>\frac{l}{r_{k}}$;

(5) $b_{j \beta} \in C^{2 l-l_{j}}(\partial \Omega)$ for each $j, \beta$ and $m_{j}<2 l, \sum_{j=1}^{l} b_{j \beta}\left(y^{\prime}\right) \sigma_{j} \neq 0$ for $|\beta|=l_{j}, y^{\prime} \in \partial G$, where $\sigma=\left(\sigma_{1}, \sigma_{2}, \ldots, \sigma_{n}\right) \in R^{n}$ is a normal to $\partial G$;

(6) for $y \in \bar{\Omega}, \xi \in R^{n}, v \in S(\varphi), \varphi \in(0, \pi),|\xi|+|v| \neq 0$ let $v+\sum_{|\alpha|=2 l} a_{\alpha}(y) \xi^{\alpha} \neq 0$;

(7) for each $y_{0} \in \partial \Omega$, the local BVP in local coordinates corresponding to $y_{0}$

$$
\begin{aligned}
& v+\sum_{|\alpha|=2 l} a_{\alpha}\left(y_{0}\right) D^{\alpha} \vartheta(y)=0, \\
& B_{j 0} \vartheta=\sum_{|\beta|=l_{j}} b_{j \beta}\left(y_{0}\right) D^{\beta} u(y)=h_{j}, \quad j=1,2, \ldots, l
\end{aligned}
$$

has a unique solution $\vartheta \in C_{0}\left(R_{+}\right)$for all $h=\left(h_{1}, h_{2}, \ldots, h_{n}\right) \in R^{n}$, and for $\xi^{\prime} \in R^{n-1}$ with $\left|\xi^{\prime}\right|+|v| \neq 0$. 
Then

(a) for all $f \in L^{\mathbf{P}}(G), \lambda \in S(\varphi)$ and sufficiently large $|\lambda|$, the problem (28)-(30) has a unique solution $u$ belonging to $W^{2 m, 2 l, \mathbf{p}}(G)$ and the following coercive uniform estimate holds:

$$
\sum_{i=0}^{2 m} s^{\frac{i}{2 m}}|\lambda|^{1-\frac{i}{2 m}}\left\|\frac{\partial^{i} u}{\partial^{i} x}\right\|_{L^{\mathbf{P}(G)}}+\sum_{|\beta|=2 l}\left\|D_{y}^{\beta} u\right\|_{L^{\mathbf{P}(G)}} \leq C\|f\|_{L^{\mathbf{P}(G)}}
$$

(b) for $\lambda=0$ the problem (28)-(30) is Fredholm in $L^{\mathbf{P}}(G)$.

Proof Let $E=L^{p_{1}}(\Omega)$. Then by virtue of [27], part (1) of Condition 1 is satisfied. Consider the operator $A$ acting in $L^{p_{1}}(\Omega)$ defined by

$$
D(A)=W^{2 l, p_{1}}\left(\Omega ; B_{j} u=0\right), \quad A u=\sum_{|\alpha| \leq 2 l} a_{\alpha}(y) D^{\alpha} u(y) .
$$

For $x \in \Omega$ also consider operators in $L^{p_{1}}(\Omega)$

$$
D\left(A_{k}\right)=W^{2 l, p_{1}}\left(\Omega ; B_{j} u=0\right), \quad A_{k}(x) u=d_{k}(x, y) u(y) .
$$

The problem (28)-(30) can be rewritten in the form (1), where $u(x)=u(x, \cdot), f(x)=f(x, \cdot)$ are functions with values in $E=L^{p_{1}}(\Omega)$. By virtue of [14, Theorem 8.2], the problem

$$
\begin{aligned}
& v u(y)+\sum_{|\alpha| \leq 2 l} a_{\alpha}(y) D_{y}^{\alpha} u(y)=f(y), \\
& B_{j} u=\sum_{|\beta| \leq l_{j}} b_{j \beta}(y) D_{y}^{\beta} u(y)=0, \quad j=1,2, \ldots, l
\end{aligned}
$$

has a unique solution for $f \in L^{p_{1}}(\Omega)$ and $\arg v \in S(\varphi),|v| \rightarrow \infty$, and the operator $A$ is $R$-positive in $L^{p_{1}}(\Omega)$, i.e., Condition 1 holds. Moreover, it is known that the embedding $W^{2 l, p_{1}}(\Omega) \subset L^{p_{1}}(\Omega)$ is compact (see, e.g., [28, Theorem 3.2.5]). Then, by using interpolation properties of Sobolev spaces (see, e.g., $[28, \$ 4]$ ), it is clear to see that condition (2) of Theorem 4 is fulfilled too. Then from Theorems 4, 5 the assertions are obtained.

\section{Systems of differential equations with VMO coefficients}

Consider the nonlocal BVPs for infinity systems of parameter-differential equations with principal VMO coefficients

$$
\begin{aligned}
& s a(x) u_{i}^{(2 m)}(x)+\sum_{k=0}^{2 m-1} \sum_{j=1}^{N} s^{\frac{k}{2 m}} b_{k i j}(x) u_{j}^{(k)}(x)+\sum_{j=1}^{N} a_{i j}(x) u_{j}(x)=f_{i}(x), \\
& x \in(0,1), i=1,2, \ldots, N, \\
& L_{k} u=\sum_{i=0}^{m_{k}} s^{\mu_{i}}\left[\alpha_{k i} u_{m}^{(l)}(0)+\beta_{k i} u_{m}^{(i)}(1)\right]=0, \quad k=1,2, \ldots, 2 m,
\end{aligned}
$$

where $s$ is a positive parameter, $a, b_{m j}, d_{m j}$ are complex-valued functions, $N$ is finite or infinite natural number, $\alpha_{k i}$ and $\beta_{k i}$ are complex numbers, $\mu_{i}=\frac{i}{2 m}+\frac{1}{2 m p}$. 
Let $a_{i j}(x)$ be a real function and

$$
\begin{aligned}
& A(x)=\left\{a_{i j}(x)\right\}, \quad u=\left\{u_{j}\right\}, \quad A u=\left\{\sum_{j=1}^{N} a_{i j}(x) u_{j}\right\}, \quad i=1,2, \ldots, N, \\
& l_{q}(A)=\left\{u: u \in l_{q},\|u\|_{l_{q}(A)}=\|A u\|_{l_{q}}=\sup _{i}\left(\sum_{j=1}^{N}\left|a_{i j}(x) u_{j}\right|^{q}\right)^{\frac{1}{q}}<\infty\right\}, \\
& x \in(0,1), 1<q<\infty .
\end{aligned}
$$

From Theorem 4, we obtain the following.

Theorem 8 Suppose the following conditions are satisfied:

(1) $a \in V M O \cap L^{\infty}(R), a(0)=a(1)$;

(2) $\left|\arg \omega_{j}-\pi\right| \leq \frac{\pi}{2}-\varphi, j=1,2, \ldots, m,\left|\arg \omega_{j}\right| \leq \frac{\pi}{2}-\varphi, j=m+1, \ldots, 2 m$ and $\frac{\lambda}{\omega_{j}} \in S(\varphi)$ for $0 \leq \varphi<\pi, \eta(x) \neq 0$ a.e. $x \in(0,1)$;

(3) $a_{i j} \neq 0$ and $a_{i j}=a_{j i}, a_{i j} \in V M O \cap L^{\infty}(R), p \in(1, \infty)$.

Then, for all $f(x)=\left\{f_{m}(x)\right\}_{1}^{N} \in L^{p}\left(0,1 ; l_{q}\right), \lambda \in S(\varphi)$ and for sufficiently large $|\lambda|$, problem (32)-(33) has a unique solution $u=\left\{u_{m}(x)\right\}_{1}^{\infty}$ belonging to $W^{2 m, p}\left((0,1), l_{q}(D), l_{q}\right)$ and the following coercive estimate holds:

$$
\sum_{i=0}^{2 m} s^{\frac{i}{2 m}}|\lambda|^{1-\frac{i}{2 m}}\left\|u^{(i)}\right\|_{L^{p}\left(0,1 ; l_{q}\right)}+\|A u\|_{L^{p}\left(0,1 ; l_{q}\right)} \leq C\|f\|_{L^{p}\left(0,1 ; l_{q}\right)} .
$$

Proof Really, let $E=l_{q}, A$ and $A_{k}(x)$ be matrices such that

$$
A=\left[d_{i j}(x)\right], \quad A_{k}(x)=\left[b_{k i j}(x) \delta_{i j}\right], \quad i, j=1,2, \ldots, N
$$

It is clear to see that the operator $A$ is $R$-positive in $l_{q}$. Therefore, by Theorem 4 , the problem (31)-(32) has a unique solution $u \in W^{2 m, p}\left((0,1) ; l_{q}(A), l_{q}\right)$ for all $f \in L^{p}\left((0,1) ; l_{q}\right)$, $\lambda \in S(\varphi)$ and the estimate (33) holds.

Remark 3 There are many positive operators in different concrete Banach spaces. Therefore, putting concrete Banach spaces and concrete positive operators (i.e., pseudodifferential operators or finite or infinite matrices for instance) instead of $E$ and $A$, respectively, by virtue of Theorems 4 and 5 , we can obtain a different class of maximal regular BVPs for partial differential or pseudo-differential equations or its finite and infinite systems with $V M O$ coefficients. 


\section{References}

1. Sarason, D: On functions of vanishing mean oscillation. Trans. Am. Math. Soc. 207, 391-405 (1975)

2. Chiarenza, F, Frasca, $M$, Longo, $P: W^{2, P}$-solvability of the Dirichlet problem for nondivergence elliptic equations with VMO coefficients. Trans. Am. Math. Soc. 336(2), 841-853 (1993)

3. Chiarenza, F: $L^{p}$ regularity for systems of PDEs with coefficients in VMO. In: Nonlinear Analysis, Function Spaces and Applications, Vol. 5 (Praque, 1994) (1994)

4. Chiarenza, F, Frasca, M, Longo, P: Interior W2,p estimates for non divergence elliptic equations with discontinuous coefficients. Ric. Mat. 40, 149-168 (1991)

5. Miranda, C: Partial Differential Equations of Elliptic Type. Springer, Berlin (1970)

6. Maugeri, A, Palagachev, DK, Softova, L: Elliptic and Parabolic Equations with Discontinuous Coefficients. Wiley, Berlin (2000)

7. Krylov, NV: Parabolic and elliptic equations with VMO coefficients. Commun. Partial Differ. Equ. 32(3), 453-475 (2007)

8. Palagachev, DK, Recke, L, Softova, L: Applications of the differential calculus to nonlinear elliptic operators with discontinuous coefficients. Math. Ann. 336(3), 617-637 (2006)

9. Ragusa, MA: Necessary and sufficient condition for VMO function. Appl. Math. Comput. 218(24), 11952-11958 (2012)

10. Ragusa, MA: Embeddings for Morrey-Lorentz spaces. J. Optim. Theory Appl. 154(2), 491-499 (2012)

11. Heck, H, Hieber, M: Maximal $L^{p}$-regularity for elliptic operators with VMO-coefficients. J. Evol. Equ. 3, 62-88 (2003)

12. Amann, H: Linear and Quasi-Linear Equations, vol. 1. Birkhäuser, Basel (1995)

13. Ashyralyev, A: On well-posedness of the nonlocal boundary value problem for elliptic equations. Numer. Funct. Anal. Optim. 24(1-2), 1-15 (2003)

14. Denk, R, Hieber, M, Prüss, J: R-boundedness, Fourier multipliers and problems of elliptic and parabolic type. Mem. Am. Math. Soc. 166, 788 (2003)

15. Favini, A, Shakhmurov, V, Yakubov, Y: Regular boundary value problems for complete second order elliptic differential-operator equations in UMD Banach spaces. Semigroup Forum 79, 1 (2009)

16. Gorbachuk, VI, Gorbachuk, ML: Boundary Value Problems for Differential-Operator Equations. Naukova Dumka, Kiev (1984)

17. Lions, JL, Peetre, J: Sur une classe d'espaces d'interpolation. Publ. Math. 19, 5-68 (1964)

18. Shakhmurov, VB: Coercive boundary value problems for regular degenerate differential-operator equations. J. Math. Anal. Appl. 292(2), 605-620 (2004)

19. Shakhmurov, VB: Separable anisotropic differential operators and applications. J. Math. Anal. Appl. 327(2), 1182-1201 (2007)

20. Shakhmurov, VB, Shahmurova, A: Nonlinear abstract boundary value problems atmospheric dispersion of pollutants. Nonlinear Anal., Real World Appl. 11, 932-951 (2010)

21. Shakhmurov, VB: Embedding theorems and maximal regular differential operator equations in Banach-valued function spaces. J. Inequal. Appl. 4, 605-620 (2005)

22. Shakhmurov, VB: Linear and nonlinear abstract equations with parameters. Nonlinear Anal., Theory Methods Appl. 73, 2383-2397 (2010)

23. Weis, L: Operator-valued Fourier multiplier theorems and maximal $L_{p}$ regularity. Math. Ann. 319, 735-758 (2001)

24. Yakubov, S, Yakubov, Y: Differential-Operator Equations. Ordinary and Partial Differential Equations. Chapman \& Hall/CRC, Boca Raton (2000)

25. John, F, Nirenberg, L: On functions of bounded mean oscillation. Commun. Pure Appl. Math. 14, 415-476 (1961)

26. Segovia, C, Torrea, JL: Vector-valued commutators and applications. Indiana Univ. Math. J. 38(4), 959-971 (1989)

27. Burkholder, DL: A geometric condition that implies the existence of certain singular integrals of Banach-space-valued functions. In: Conference on Harmonic Analysis in Honor of Antoni Zygmund, Vol. I, II, Chicago, III., 1981, pp. 270-286. Wadsworth, Belmont (1983)

28. Triebel, H: Interpolation Theory, Function Spaces, Differential Operators. North-Holland, Amsterdam (1978)

29. Blasco, O: Operator valued BMO commutators. Publ. Mat. 53(1), 231-244 (2009)

30. Hytönen, T, Weis, L: A T1 theorem for integral transformations with operator-valued kernel. J. Reine Angew. Math. 599, 155-200 (2006)

31. Grisvard, P: Elliptic Problems in Nonsmooth Domains. Pitman, London (1985)

32. Besov, OV, Ilin, VP, Nikolskii, SM: Integral Representations of Functions and Embedding Theorems. Nauka, Moscow (1975)

doi:10.1186/1687-1812-2013-42

Cite this article as: Shakhmurov: Optimal regular differential operators with variable coefficients and applications. Fixed Point Theory and Applications 2013 2013:42. 